\title{
RECOVARI - Efficacy and Safety of Massage on Surgical Stress in Colorectal Cancer Patients: Study Protocol for A Randomized Controlled, Three-Arm Parallel Group, Clinical Trial
}

Paul Georg Werthmann ( $\square$ paul.werthmann@ifaemm.de )

Universitätsklinikum Freiburg https://orcid.org/0000-0002-1808-7787

Dirk Cysarz

Universitat Witten/Herdecke

Johannes Naumann

European Insitute for Physical Therapy and Balneology

Monique van Dijk

Erasmus Medical Centre: Erasmus MC

Roman Huber

Albert-Ludwigs-Universität Freiburg: Albert-Ludwigs-Universitat Freiburg

Ann-Kathrin Lederer

University Medical Center Freiburg: Universitatsklinikum Freiburg

Gunver Sophia Kienle

University Medical Center Freiburg: Universitatsklinikum Freiburg

Research Article

Keywords: Rhythmical Embrocations, Massage, Stress, Heart-Rate-Variability, Colorectal cancer.

Posted Date: November 11th, 2021

DOI: https://doi.org/10.21203/rs.3.rs-764228/v1

License: (a) (i) This work is licensed under a Creative Commons Attribution 4.0 International License. Read Full License 


\section{Abstract}

- Background: Patients' physiological stress reactions to surgery have been ameliorated by refining surgical techniques, and improving pain therapy. Moreover, another way to reduce stress could be the use of Rhythmical embrocation (RE), a type of bodily massage applied with soft strokes, which has been shown to reduce the subject's pulse rate and blood pressure. This trial will investigate whether RE is effective to reduce stress, and what effects it exerts on sleep quality, nausea and vomiting, pain, medication consumption, mood, mobility, and length of hospital. Furthermore, the occurrence of complications associated with RE will be established.

- Methods: A randomized controlled, three-arm parallel group trial will include 60 patients with colorectal cancer scheduled for surgery, randomized into three different intervention groups: (1) RE performed by professionals, (2) RE performed by students; and (3) empathic conversation. The intervention will be performed twice daily over four consecutive postoperative days. Before and after the intervention days, heart rate variability (primary outcome) will be established overnight as a measure of stress. The patients will fill out questionnaires on pain, sleep, nausea/vomiting and well-being. Possible complications from RE will be recorded daily. Vital signs, medication and surgery data will be retrieved from the patient record. Nursing students will be interviewed about job satisfaction, empathy and their identification with RE.

- Discussion: Massage has been shown to have stress relieving effects. However, it's effectiveness in clinical settings is rarely investigated. With the postoperative setting we investigate massage in a situation of high physiological stress. Reduction of stress perioperatively has shown to reduce complications and improve recovery. With heart rate variability we include a physiologic, objective parameter to supplement them against the questionnaire responses of patients. Massage might reduce stress in stressful situations in healthcare and contribute to a better outcome of these patients.

Trial registration: The trial has been registered in the German Clinical Trials Register (DRKS) under the ID DRKS00023407 on $2^{\text {nd }}$ November 2020. The trial was approved by the ethics committee of the University Medical Center Freiburg under the number $356 / 20$ on $20^{\text {th }}$ October 2020.

\section{Introduction}

\section{Background and rationale $\{6 a\}$}

Stress in humans may lead to insomnia, increased pain perception, loss of concentration and memory, and worsening of immunologic functions [1]. Stress is also associated with increased use of medication such as tranquilizers, sleep medication or pain medication - and consequently a higher risk of medication abuse, dependency and polypharmacy [2]. New and innovative methods to reduce stress and its related conditions are being explored. Stress reduction methods preferably are effective without the risk of complications. 
Stress responses of the body can be measured through activation of the sympathetic nervous system, pituitary hormone secretion, insulin resistance, immunological and hematological changes, as well as heart rate variability (HRV) [3, 4]. Standards have been developed for HRV measurements [5], and HRV is increasingly being used in stress research $[4,6-18]$. Because stress might negatively influence the recovery from surgery, it is important to find ways to reduce it [19].

This research project aims at reducing patients' stress after colorectal cancer surgery. Colorectal cancer is the 4th most commonly diagnosed cancer after breast, lung and prostate cancer [20]. About $80 \%$ of colorectal cancers present in a localized stage in which surgery can be curative. The postoperative course is often associated with pain, anxiety, nausea/vomiting, and obstipation. Introducing methods to reduce stress could be beneficial. One such method is the rhythmical embrocations (RE) manual massage therapy. This therapy was developed at the beginning of the $20^{\text {th }}$ century by the physicians Dr. Wegman und Dr. Hauschka [21]. RE-therapy makes use of oils or emulsions which the nurse massages gently in circular or linear strokes over the body [21, 22]. This type of massage seems to have relaxing effects, as reflected by pulse rate reduction and lowering of blood pressure, as well as pain reduction and mood improvement in patients with chronic pain $[23,24]$.

\section{Objectives $\{7\}$}

The primary objective of this trial is to establish if RE-therapy results in changes of stress levels in postsurgical colorectal cancer patients as measured with HRV.

Secondary objectives are to explore the effect of RE on sleep quality, nausea and vomiting, pain, medication, mood, grade of mobilization, length of stay in the hospital, as well as to explore complications associated with the intervention.

Furthermore, we investigate whether students show altered empathy and job satisfaction when they are providing RE in this trial, and whether they feel an inner correspondence with carrying out RE.

\section{Trial design $\{8\}$}

The trial is designed as a mono-center, randomized controlled, open-label, three-arm parallel group trial. Patients will be allocated 1:1:1 to the three arms.

\section{Methods: Participants, Interventions And Outcomes Study setting $\{9\}$}

Participants will be recruited from the department of General and Visceral Surgery of the University Medical Center Freiburg in Germany.

\section{Eligibility criteria $\{10\}$}


Eligible patients are those diagnosed with colorectal cancer and scheduled for surgery. Inclusion criteria are as follows: patients in an inpatient setting after colorectal cancer surgery; able to fill out questionnaires or answer questions; age from 18 years onwards; legal competence; ability to understand the nature of the trial and the trial-related procedures and to comply with them; ability to understand German language and having provided written informed consent obtained according to international guidelines and local laws. Exclusion criteria are: severe surgical complication; severe cardiac complication; sepsis; cardiac diseases which interfere with the HRV analysis, such as severe arrhythmias or a pacemaker; severe psychiatric disorder; pregnancy or breastfeeding; clinically significant comorbidity critically influencing the patient to adhere to the protocol; hypersensitivity to skincare products; skin conditions in the area of the back or the feet; isolated nursing; participation in a clinical trial within the 3 months before the start of this study or simultaneously - except studies which are not investigating therapeutic or diagnostic procedures and are not interfering with the current study.

\section{Who will take informed consent? \{26a\}}

During the pre-surgical visit, eligible patients will be informed about the trial by the surgeons. The patients will be informed that participation is voluntary and that no disadvantages will result from non-

participation. If patients are interested in participating in the trial, printed patient information will be handed out to them. After having read the patient information, the patients will have the opportunity to ask questions and to decide about their consent. Written informed consent will be obtained by the doctors.

\section{Additional consent provisions for collection and use of participant data and biological specimens $\{26 \mathrm{~b}\}$}

Not applicable.

\section{Interventions}

\section{Explanation for the choice of comparators $\{6 \mathrm{~b}\}$}

To differentiate between the pure effect of massage and that of concomitant empathic attention, the control arm involves merely an empathic conversation.

\section{Intervention description $\{11$ a $\}$}

Patients allocated to two arms will receive RE-therapy - in one arm performed by RE-professionals and in one arm by nursing students. Patients in the third arm will receive empathic conversations with a nursing student. Nursing students (as an alternative: nursing assistants or medical students) will be trained in RE in three teaching units of $3 \frac{1}{2}$ hours each. The training will be carried out by two professionals certified in RE by the International Forum for Anthroposophic Nursing [25] or having trained in a standardized REcurriculum and having working experience with RE-therapy with patients for at least 5 years. 
RE will be applied as described in the RE-literature and taught in RE-training courses (for a detailed description see table 1) [26].

Intervention

Participants will receive RE of the back and the feet with the application of $2 \mathrm{~mL}$ pure almond oil in pharmacopoeial [27] quality for circa 10 minutes between 8 and 10 am and between 6 and $10 \mathrm{pm}$. The intensity of RE of the back will vary between $1-3$, and that of the feet between $1-5$ on a scale of $1=$ 'softest massage possible' to $10=$ 'very intense massage'.

The control intervention will consist of a 10-minute empathic conversation in the same time windows. The conversation will start with an open question, such as "How are you today?" and then takes a spontaneous course. A list with proposed questions and topics is provided to the students.

All patients will be asked to rest for 20 minutes after the intervention. Staff and patients in the same room will be told about the intervention and resting time and will be asked not to interrupt during these periods. In addition, a sign will be put up on the door signaling 'study intervention, please do not disturb until x: xx'.

\section{Criteria for discontinuing or modifying allocated interventions $\{11 \mathrm{~b}\}$}

All participants will be informed that participation in the trial is voluntary, and can be stopped at any time. If a complication associated with the intervention occurs, the principal investigator judges if this necessitates discontinuation of the intervention. After the intervention has been stopped, no other intervention will be given.

\section{Strategies to improve adherence to interventions $\{11 \mathrm{c}\}$}

Every intervention is applied and monitored by the study personnel. The intervention is applied and most outcomes are assessed in hospital and supported by the patient's care team. Therefore high adherence is expected and no further specific strategy implemented.

\section{Relevant concomitant care permitted or prohibited during the trial $\{11 d\}$}

All study interventions are added to the usual care that participants receive. No care is prohibited.

\section{Provisions for post-trial care $\{30\}$}

After completion, the study participants will receive standard care during hospitalization and after discharge.

\section{Outcomes $\{12\}$}

The primary outcome is level of stress as measured with HRV on day 5 overnight from the whole sleeping time as defined from the HRV and movement data as recorded by the HRV device. 
The secondary outcomes are sleep quality, nausea and vomiting, pain intensity, analgesics consumption, sedatives consumption, emetics consumption, mood, grade of mobilization, length of stay in hospital, complications associated with the RE-therapy. Nursing students will be asked regularly about job satisfaction (UWES), empathy and motivation to carry out the RE.

\section{Table 1: Participant timeline $\{13\}$}




\begin{tabular}{|c|c|c|c|c|c|c|c|c|c|}
\hline PERIODS & & & Baseline & Inter & ention & & & & Follow- \\
\hline VISITS & $\begin{array}{l}\text { Screening } \\
\text { / Consent }\end{array}$ & $\begin{array}{l}\text { Surgery } \\
\text { I ICU }\end{array}$ & $\begin{array}{l}\text { Visit } 1 \\
\text { (Baseline) }\end{array}$ & & $\begin{array}{l}\text { Visit } \\
2\end{array}$ & & & $\begin{array}{l}\text { Final } \\
\text { Visit }\end{array}$ & \\
\hline & & & & & & & & EOT & \\
\hline Time & $\begin{array}{l}\text { Day - } 1 \\
\text { (day } \\
\text { before } \\
\text { surgery) }\end{array}$ & $\begin{array}{l}\text { Day X- } \\
Y\end{array}$ & $\begin{array}{l}\text { Day } 1 \\
\text { (arrival on } \\
\text { regular } \\
\text { ward) }\end{array}$ & $\begin{array}{l}\text { Day } \\
2\end{array}$ & $\begin{array}{l}\text { Day } \\
3\end{array}$ & $\begin{array}{l}\text { Day } \\
4\end{array}$ & $\begin{array}{l}\text { Day } \\
5\end{array}$ & $\begin{array}{l}\text { Day } \\
6\end{array}$ & \\
\hline $\begin{array}{l}\text { Informed } \\
\text { consent }\end{array}$ & $X$ & & & & & & & & \\
\hline $\begin{array}{l}\text { Inclusion / } \\
\text { Exclusion } \\
\text { criteria, } \\
\text { demographic } \\
\text { data }\end{array}$ & $x$ & & $(X)^{\star}$ & & & & & & \\
\hline $\begin{array}{l}\text { Medical } \\
\text { history, surgery } \\
\text { type / length / } \\
\text { complications }\end{array}$ & & & $x$ & & & & & $(X)^{\star}$ & \\
\hline $\begin{array}{l}\text { Sleeping } \\
\text { habits, } \\
\text { movement } \\
\text { habits, } \\
\text { stress factors }\end{array}$ & $x$ & & & & & & & & \\
\hline Randomization & & & $x$ & & & & & & \\
\hline $\begin{array}{l}\text { Vital signs (RR, } \\
\text { Temp., bowel } \\
\text { activity) }\end{array}$ & & & $x$ & $X, X$ & $X, X$ & $X, X$ & $X, X$ & $x$ & \\
\hline $\begin{array}{l}\text { Mobility } \\
\text { (EBoMo) }\end{array}$ & & & $\mathrm{X}$ & & & & & $x$ & \\
\hline 24-ECG (HRV) & & & $x$ & & & & $x$ & & \\
\hline $\begin{array}{l}\text { Intervention } \\
\text { morning }\end{array}$ & & & & $x$ & $x$ & $x$ & $x$ & & \\
\hline $\begin{array}{l}\text { Intervention } \\
\text { evening }\end{array}$ & & & & $x$ & $x$ & $x$ & $x$ & & \\
\hline Pain (VAS) & & & $x$ & & $x$ & & & $x$ & \\
\hline
\end{tabular}

Abbreviations: ICU: intensive care unit; EOT: end of trial; RR: blood pressure; EBoMo: Evaluationsbogen Mobilität - evaluation sheet mobility; ECG: electrocardiogram; HRV: heart rate variability; VAS: visual analogue scale; RCSQ: Richards Campbell sleep questionnaire; MDBF: Mehrdimensionaler Befindlichkeitsfragebogen - multidimensional mood questionnaire; RE: rhythmical embrocations. *Inclusion and exclusion criteria will be checked again on day 1 . 


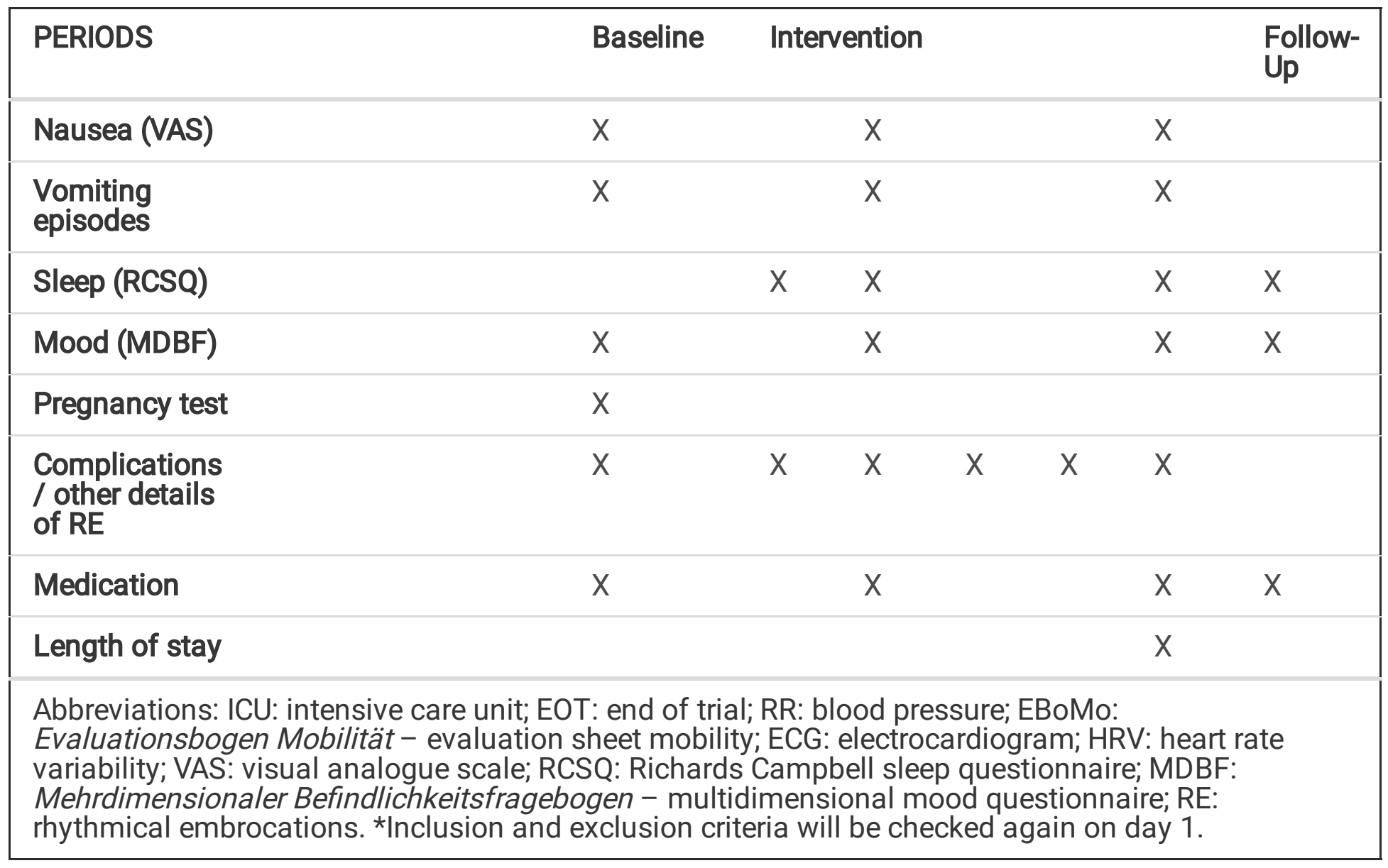

\section{Sample size $\{14\}$}

The sample size calculation is based on the data of a previous trial investigating perioperative stress with heart rate variability as outcome measure. In that trial, significant changes in the SDNN had been recorded at different perioperative time points [6]. The preoperative SDNN was 116 milliseconds (ms); on day one after surgery it was $65 \mathrm{~ms}$, and on postoperative day seven it was $87 \mathrm{~ms}$ (values are approximate values as the concrete values are not given in the article text, but only displayed as a bar chart; because the authors did not answer to our request, we converted the bar chart into numbers using WebPlotDigitizer [28]). Based on these figures, we presume the median SDNN in the conversation group to be $80 \mathrm{~ms}$ on postoperative day 5 . In the intervention groups, we presume a faster recovery of the SDNN; i.e., $110 \mathrm{~ms}$ on day 5 (therefore we set $\delta=30$ ). In the previous trial, the standard deviation was around $30(\sigma=30)$ for all measurements. Based on a t-distribution, a two-tailed $\alpha=0.05$ and a power $(\beta)$ of 0.8 , we calculated a sample size of 17 patients per group, and thus 51 in total. To adjust for attrition, we will enroll 60 patients.

\section{Recruitment \{15\}}

In the surgical department 120 patients are operated on each year who match the inclusion criteria of our study. We therefore consider a recruitment rate of 60 in 12 months to be feasible. We expect a small dropout rate because data collection encompasses five days only and eligible patients are already hospitalized. 
Assignment of interventions: allocation

\section{Sequence generation $\{16 a\}$}

For allocation to the various trial arms, a computer-generated list of random numbers will be used. The list will be retrieved by an independent researcher only and randomization will be performed from this list for each individual patient after inclusion in the study.

\section{Concealment mechanism $\{16 \mathrm{~b}\}$}

The allocation sequence is generated by an independent researcher using the website Randomization.com [29]. No stratification or block randomization is used.

\section{Implementation $\{16 \mathrm{c}\}$}

The participants are enrolled by a doctor at the pre-surgical visit. The inclusion and exclusion criteria are checked again after surgery at arrival on the normal ward. After inclusion of a new patient, the investigator and the trial assistant are informed. The investigator or the trial assistant sends the sequential number of the new patient electronically to the independent researcher, upon which the independent researcher sends back the code of intervention from the allocation list.

\section{Assignment of interventions: Blinding}

\section{Who will be blinded $\{17 a\}$}

All study staff who are directly involved in the intervention (RE experts, nursing students) cannot be blinded. Study stuff providing RE are instructed not to inform the patients if they are treated by experts or students. The patients are informed in the patient information, that they will not be told if they are treated bey experts or students in the intervention arms with RE. Data collection and data analysis will be blinded.

\section{Procedure for unblinding if needed $\{17 \mathrm{~b}\}$}

Not applicable

\section{Data collection and management}

\section{Plans for assessment and collection of outcomes $\{18 \mathrm{a}\}$}

The primary outcome 'level of stress' will be assessed from the heart rate variability (HRV), which will be recorded on days 1 and 5 overnight using a one-channel electrocardiogram (ECG, Bittium Faros 180 [30]). Data will be processed according to the 1996 guideline of the Task Force of The European Society of Cardiology and The North American Society of Pacing and Electrophysiology [5]. 
The secondary outcomes will be assessed as follows: Sleep quality with the Richards-Campbell sleep questionnaire (RCSQ) in its German validated translation [31] on days 2, 3 and 6 . The severity of nausea within the last 24 hours will be assessed using a $100 \mathrm{~mm}$ visual analogue scale (VAS, from: no nausea to: worst imaginable nausea) [32,33] on days 1,3 and 6 . Number of vomiting episodes within the last 24 hours will be documented [32] on days 1, 3 and 6 . Pain within the last 24 hours will be assessed on days 1,3 and 6 using a 100 mm VAS (from: no pain to: strongest imaginable pain). Consumption of analgesics, sedatives, emetics and other relevant medication will be retrieved from the patient's chart. Mood will be assessed on days 1, 3 and 6 using the Mehrdimensionaler Befindlichkeitsfragebogen (MDBF, Multidimensional well-being questionnaire, a validated questionnaire measuring well-being in the dimensions of pleasant - unpleasant, awake - sleepy, and calm - restless [34]). For the assessment of activity level, the Evaluationsbogen Mobilität (EBoMo, mobility evaluation sheet, a simple scoring tool for activity level e.g. in nursing homes [35]) will be scored at baseline and on day 6 .

Length of stay in the hospital will be recorded as indicated in the patient's chart. Regarding complications associated with the intervention, patients will be asked to report these before the evening treatment.

The RCSQ and MDBF will be mailed to the participants 1 month after discharge. They will be telephoned and asked to fill out the questionnaires and send them back to the study center in the pre-paid envelope. In addition, they will also be asked to indicate current medication consumption, especially sleep medication and tranquilizing drugs. At the start and end of the trial and every 3 months during the trial, the nursing students will be asked to fill out the following validated questionnaires: Utrecht Work Engagement Scale - Short form (UWES-9) [36], Interpersonal Reactivity Index (IRI) [37]. At the end of their work in the trial they will fill out the questionnaire: Inner Correspondence and feelings of Peaceful Relief (ICPH) [38].

\section{Plans to promote participant retention and complete follow-up $\{18 \mathrm{~b}\}$}

Regarding the short time for each participant in the trial, we expect we do not have to promote participant retention or collect outcome data for participants who discontinue or deviate from intervention protocols.

\section{Data management $\{19\}$}

HRV data will be extracted and analyzed using Matlab (The Mathworks) [39].

Data of questionnaires and open questions will be collected on paper in prepared questionnaires and data sheets. This data will be collected and managed using REDCap (Research Electronic Data Capture) tools hosted at the University Medical Center Freiburg [40]. REDCap is a secure, web-based application designed to support data capture for research studies, providing: 1) an intuitive interface for validated data entry; 2) audit trails for tracking data manipulation and export procedures; 3 ) automated export procedures for seamless data downloads to common statistical packages; and 4) procedures for importing data from external sources. The data will be entered into REDCap 10.0.28 into fields with prespecified data ranges and plausibility checks. 
The datasets from Matlab and REDCap will then be merged by patient id.

\section{Confidentiality $\{27\}$}

All patient data is collected and processed pseudonymously. The ID list with the patients' names will only be available to the trial investigator. The trial data is only accessible to the trial investigator and authorized personnel.

\section{Plans for collection, laboratory evaluation and storage of biological specimens for genetic or molecular analysis in this trial/future use $\{33\}$}

Not applicable

\section{Statistical methods}

\section{Statistical methods for primary and secondary outcomes $\{20 \mathrm{a}\}$}

Data analysis will be performed using R 4.0.3 (or higher) [41]. Data-analysis will be performed with an intention-to-treat approach using the full analysis set. A p-value $<0.05$ will be regarded as indicating significance.

For the primary outcome 'level of stress', the SDNN will be calculated from HRV during sleep from the recording of day 5 ; the mean and standard deviation of each intervention group will be calculated. The following testing sequence will then be performed: the mean with standard deviation of the control group (empathic conversation) will be compared with mean of the expert RE group using the T-test; if the null hypothesis (no difference between the groups) can be rejected, the control group will be compared to the student RE group; if the null hypotheses can be rejected here as well the expert RE group will be compared with the student RE group.

As secondary analysis, analysis of covariance (ANCOVA) will be performed with SDNN at day 5 and group as variable and SDNN at baseline as a covariable, if preconditions for ANCOVA are fulfilled. Cohen's $d$ will be calculated between 2 of the 3 groups and a Cohen's $d>0.5$ will be considered a medium effect size. If parametric tests are not possible, non-parametric tests will be performed and the median with the 25th and 75th percentile reported.

For the other secondary outcomes, the same procedures will be performed and for categorical variables the chi-square test will be used. The paired T-test will be performed in continuous variables to compare characteristics of one group between two time points.

\section{Interim analyses $\{21 b\}$}

No interim analyses are planned. 
The coordinating investigator is under obligation to monitor the progress of the clinical trial with regard to safety-relevant developments and, if necessary, initiate the premature termination of a treatment arm or the entire clinical trial.

A treatment arm or the entire clinical trial will be terminated prematurely if:

- the coordinating investigator considers that the termination of the trial is necessary,

- indications arise that the trial patients' safety is no longer guaranteed,

- an insufficient recruitment rate makes a successful conclusion of the clinical trial unrealizable/no longer feasible.

If the clinical trial is prematurely terminated, the ethics committee will be informed by the coordinating investigator.

The trial participant can withdraw consent for trial participation at any time, without having to give reasons.

The responsible investigator may only withdraw a patient from participation in the trial for the following reasons: extreme circumstances arise which make any trial-relevant follow-up impossible.

The documentation should be completed as far as possible under these circumstances, e.g. a final examination and documentation according to the protocol (if possible), a documentation of the premature trial termination on the CRF and in the medical record, giving reasons, should be ensured.

\section{Methods for additional analyses (e.g. subgroup analyses) \{20b\}}

Subgroup analyses will be performed regarding possible confounders such as type of surgery or tumor stage, as these might impact participants' stress levels.

\section{Methods in analysis to handle protocol non-adherence and any statistical methods to handle missing data $\{20 \mathrm{c}\}$}

Missing data will be imputed by full-information maximum likelihood method. The per-protocol group will be defined as having no missing data in the relevant outcome and having participated in more than 5 of 8 treatments.

\section{Plans to give access to the full protocol, participant level-data and statistical code $\{31 \mathrm{c}\}$}

The full protocol is available as additional content to this article. Because of privacy reasons, the full dataset will not be made publicly available.

Oversight and monitoring

Composition of the coordinating center and trial steering committee $\{5 \mathrm{~d}\}$ 
Principal Investigator (PGW) and deputy (GSK):

- Design of the trial

Preparation of protocol and revisions

- Publication of study reports

- Agreement of final protocol

Department of General and Visceral Surgery

- Recruitment of colorectal cancer patients

Team of RE experts

- Organization of patient interventions

- Training of students in RE

Composition of the data monitoring committee, its role and reporting structure $\{21 \mathrm{a}\}$

As the possible harm of the intervention is presumed to be small, no data monitoring committee is planned for this trial.

\section{Adverse event reporting and harms $\{22\}$}

Complications possibly associated with the intervention will be recorded on a daily basis. Severe complications will be reported immediately to the principal investigator, who then decides about the continuation or discontinuation of the intervention.

Frequency and plans for auditing trial conduct $\{23\}$

No auditing is planned

Plans for communicating important protocol amendments to relevant parties (e.g. trial participants, ethical committees) $\{25\}$

The investigator will report every important protocol modification to the ethics committee of the University Medical Center Freiburg, to the German trials Registry and the respective study staff.

\section{Dissemination plans $\{31$ a $\}$}

The investigator will prepare a publication of the results of the study and submit it to a peer-reviewed journal. There are no publication restrictions.

\section{Discussion}


The effect of human touch is increasingly investigated in psychological and medical research. Especially the beneficial effects of social and therapeutic touch on stress levels have been studied, which has led to the discovery of C-tactile afferents that have special properties to mediate tactile stimuli from another person and calming effects of touch [42-45]. Massage has shown to be effective in clinical settings of pain and conditions of the musculoskeletal system [46-49]. In surgical settings, massage before surgical procedures has been associated with anxiety reduction [50,51]. And massage after cardiac surgery reduced pain, anxiety and tension [52]. In further clinical situations it is mostly unknown if massage has a robust clinical benefit for patients. To the best of our knowledge, this is the first randomized controlled trial on RE and also the first randomized controlled trial on massage after colorectal surgery investigating the physiologic parameter HRV. With this trial, the impact of massage is investigated in a clinical relevant stress situation. In addition to validated patient questionnaires on clinical significant issues, the effects on stress are also evaluated with HRV as a physiologic parameter which is not prone to biases of questionnaires like courtesy responses. Blinding in massage trials is especially difficult, if the control intervention consists of an activity without touch. However, we choose this type of control intervention in concordance with other authors to compare the effectiveness of a specific massage with empathic attention without touch.

As a limitation it could be discussed, that the primary endpoint HRV is not a clinically relevant outcome parameter and rather endpoints like frequency and severity of complications after surgery or even cancer relapse should be used. However, improvement of HRV through massage has been shown in different clinical settings [53-56] and is associated with complications after surgery [57] and the course of cancer patients [58]. Therefore, we see HRV as a meaningful parameter to investigate in this setting.

Furthermore, we have added clinically highly significant outcomes like pain, nausea and vomiting, mobilization, length of stay in hospital and validated and frequently used questionnaires. Therefore, we regard our outcomes as reliable and clinically meaningful.

With the trial we also realize practical issues of implementing massage therapy into the clinical practice of a university hospital, where the working days of caregivers are stressful and patients are partly overwhelmed by their new diagnosis and the hospital environment. For this setting we also set up the third arm of the study with students carrying out the massage - which might be a future perspective of implementing massage into the clinical setting. Students usually have less stress and more time to empathically carry out such interventions. As massage also might have an effect on the professional providing them [59-61], we also investigate the effect of this intervention on the providing students regarding their job satisfaction, empathy and identification with the intervention.

\section{Trial status}

The trial is currently carried out under the protocol version 1.2. In January 2021 the first patient was recruited and we have currently 24 patients included in the trial. Recruitment is planned until the end of 
November 2021. To increase recruiting we are currently planning to change to a multicenter setting and include another trial center.

\section{Abbreviations}

ANCOVA - analysis of covariance

EBoMo - Evaulationsbogen Mobilität (mobility evaluation sheet)

ECG - electrocardiogram

HRV - heart rate variability

ICPH - Inner Correspondence and feelings of Peaceful Relief

IRI - Interpersonal Reactivity Index

MDBF - Mehrdimensionaler Befindlichkeitsfragebogen (Multidimensional well-being questionnaire)

RCSQ - Richards-Campbell sleep questionnaire

RE - Rhythmical Embrocations according to Wegman/Hauschka

SDNN - standard deviation of the NN interval

UWES-9 - Utrecht Work Engagement Scale - Short form

VAS - visual analogue scale

\section{Declarations}

\section{Acknowledgements}

We would like to thank Dr. Göran Köber (Institute of Medical Biometry and Statistics, University Hospital Freiburg) for advice about the statistical analysis.

The trial is a research project of PGW in the master's program Clinical Epidemiology at Erasmus University Rotterdam, the Netherlands and is supervised by Prof. Monique van Dijk (Erasmus University Medical Center, Rotterdam, the Netherlands).

\section{Authors' contributions $\{31 \mathrm{~b}\}$}

PGW is the principal author of the article and the study protocol, prepared the draft versions and included suggested changes into the manuscript. JN provided substantial parts of the statistical methods used and revised the text. DC revised the text and contributed to the accuracy of the parts on heart rate 
variability. $\mathrm{RH}$ revised the protocol and the article and gave substantial advice on practical and organizational issues. MvD gave advice on the trial design and revised several protocol versions and the article. AKL provided knowledge about the surgical procedures and the procedures of the surgical ward, gave input for the trial protocol and revised the article. GSK gave substantial input for the trial design, revised several protocol versions and the article and is supervisor of the trial. All authors read and approved the final version of the manuscript.

\section{Funding $\{4\}$}

The trial is funded by the Dr. Hauschka Stiftung, Bad Boll / Eckwälden, Germany, the Christophorus Stiftung, Stuttgart Germany and the Software AG - Stiftung, Darmstadt, Germany. The funding has no influence on the design, conduct, analysis interpretation and publication of the study.

The article processing charge was funded by the Baden-Württemberg Ministry of Science, Research and Art and the University of Freiburg in the funding program Open Access Publishing.

\section{Availability of data and materials $\{29\}$}

The principal investigator has full authority over the final dataset.

\section{Ethics approval and consent to participate $\{24\}$}

Ethical Review Board of the University Medical Center Freiburg 356/20, received on $20^{\text {th }}$ October 2020. Written, informed consent to participate will be obtained from all participants.

\section{Consent for publication $\{32\}$}

Not applicable

\section{Competing interests $\{28\}$}

The authors declare that they have no competing interests.

\section{References}

1. Yaribeygi H, Panahi Y, Sahraei H, Johnston TP, Sahebkar A. The impact of stress on body function: A review. EXCLI J. 2017;16:1057-72.

2. Sinha R. Chronic Stress, Drug Use, and Vulnerability to Addiction. Ann N Y Acad Sci. 2008;1141:10530. doi:10.1196/annals.1441.030.

3. Desborough JP. The stress response to trauma and surgery. Br J Anaesth. 2000;85:109-17. doi:10.1093/bja/85.1.109.

4. Ernst G. Heart Rate Variability. London: Springer London; 2014. doi:10.1007/978-1-4471-4309-3. 
5. Malik M, Bigger JT, Camm AJ, Kleiger RE, Malliani A, Moss AJ, et al. Heart rate variability: Standards of measurement, physiological interpretation, and clinical use. Eur Heart J. 1996;17:354-81. doi:10.1093/oxfordjournals.eurheartj.a014868.

6. Ushiyama T, Nakatsu T, Yamane S, Tokutake H, Wakabayashi H, Ishimura K, et al. Heart Rate Variability for Evaluating Surgical Stress and Development of Postoperative Complications. Clin Exp Hypertens. 2008;30:45-55. doi:10.1080/10641960701813908.

7. Amalan S, Vaishali B, S.P. P, Joseph J, Sivaprakasam M. Pre-surgery Stress Monitoring Using Heart Rate Variability Measures. In: 2019 41st Annual International Conference of the IEEE Engineering in Medicine and Biology Society (EMBC). Berlin, Germany: IEEE; 2019. p. 4592-5. doi:10.1109/EMBC.2019.8856409.

8. Borghi-Silva A, Reis M, Mendes R, Melo R, Pantoni C, Quitério R, et al. Heart rate variability in the elderly with chronic obstructive pulmonary disease submitted to acute application of bi-level positive airway pressure. Crit Care. 2005;9 Suppl 2:P87. doi:10.1186/cc3631.

9. Jarczok MN, Koenig J, Wittling A, Fischer JE, Thayer JF. First Evaluation of an Index of Low VagallyMediated Heart Rate Variability as a Marker of Health Risks in Human Adults: Proof of Concept. J Clin Med. 2019;8:1940. doi:10.3390/jcm8111940.

10. Yeh GY, Mietus JE, Peng C-K, Phillips RS, Davis RB, Wayne PM, et al. Enhancement of sleep stability with Tai Chi exercise in chronic heart failure: preliminary findings using an ECG-based spectrogram method. Sleep Med. 2008;9:527-36.

11. Powezka K, Adjei T, von Rosenberg W, Normahani P, Goverdovsky V, Standfield NJ, et al. A pilot study of preoperative heart rate variability predicting pain during local anesthetic varicose vein surgery. $J$ Vasc Surg Venous Lymphat Disord. 2019;7:382-6. doi:10.1016/j.jvsv.2018.08.008.

12. Tan C, Saito N, Miyawaki I. Changes in Heart Rate and Autonomic Nervous Activity after Orthopedic Surgery in Elderly Japanese Patients. Kobe J Med Sci. 2017;62:E129-35.

13. Miliauskas P, Zemaityte D, Varoneckas G, Zurauskas A, Tikuisis R. [Diagnostic value of heart rate variability in general anesthesia]. Med Kaunas Lith. 2002;38 Suppl 2:97-100.

14. Ledowski T, Stein J, Albus S, MacDonald B. The influence of age and sex on the relationship between heart rate variability, haemodynamic variables and subjective measures of acute post-operative pain. Eur J Anaesthesiol. 2011;28:433-7. doi:10.1097/EJA.0b013e328343d524.

15. Ushiyama T, Mizushige K, Wakabayashi H, Nakatsu T, Ishimura K, Tsuboi Y, et al. Analysis of heart rate variability as an index of noncardiac surgical stress. Heart Vessels. 2008;23:53-9. doi:10.1007/s00380-007-0997-6.

16. Scheffler P, Muccio S, Egiziano G, Doonan RJ, Yu A, Carli F, et al. Heart Rate Variability Exhibits Complication-Dependent Changes Postsurgery. Angiology. 2013;64:597-603. doi:10.1177/0003319712461932.

17. Yung MC, Chang Y, Lai ST, Tsou MY, Chan KH. Improved postoperative pain relief via preemptive analgesia in relation to heart rate variability for coronary artery bypass grafting: a preliminary report. Zhonghua Yi Xue Za Zhi Chin Med J Free China Ed. 1997;60:28-35. 
18. Spellenberg C, Heusser P, Büssing A, Savelsbergh A, Cysarz D. Binary symbolic dynamics analysis to detect stress-associated changes of nonstationary heart rate variability. Sci Rep. 2020;10:15440. doi:10.1038/s41598-020-72034-2.

19. Helander EM, Webb MP, Menard B, Prabhakar A, Helmstetter J, Cornett EM, et al. Metabolic and the Surgical Stress Response Considerations to Improve Postoperative Recovery. Curr Pain Headache Rep. 2019;23. doi:10.1007/s11916-019-0770-4.

20. Cancer of the Colon and Rectum - Cancer Stat Facts. SEER. https://seer.cancer.gov/statfacts/html/colorect.html. Accessed 17 Dec 2019.

21. Layer M. Sich auf der Erde Zuhause fühlen. In: Bertram M, Kolbe HJ, editors. Dimensionen therapeutischer Prozesse in der Integrativen Medizin. Wiesbaden: Springer Fachmedien Wiesbaden; 2016. p. 155-68. doi:10.1007/978-3-658-12149-5_10.

22. Anthroposophische Pflegepraxis: Grundlagen und Anregungen für alltägliches Handeln. Berlin: Salumed-Verlag; 2017.

23. Ostermann T, Blaser G, Bertram M, Michalsen A, Matthiessen PF, Kraft K. Effects of Rhythmic Embrocation Therapy With Solum Oil in Chronic Pain Patients: A Prospective Observational Study. Clin J Pain. 2008;24:237-43. doi:10.1097/AJP.0b013e3181602143.

24. Bertram M. Rhythmische Einreibungen nach Wegman/Hauschka. In: Bertram M, Kolbe HJ, editors. Dimensionen therapeutischer Prozesse in der Integrativen Medizin. Wiesbaden: Springer Fachmedien Wiesbaden; 2016. p. 107-22. doi:10.1007/978-3-658-12149-5_7.

25. International Forum for Anthroposophic Nursing, editor. Handbook for the Certification of Specialists in Rhythmical Einreibungen according to Wegman/Hauschka (IFAN). 4th edition. Dornach/Switzerland; 2019. https://www.qualitaet-ap.org/index.php?action=downloadview\&file $=100$.

26. Batschko E-M. Einführung in die rhythmischen Einreibungen: nach Wegman/Hauschka. Stuttgart: Mayer; 2011.

27. Deutscher Apotheker-Verlag Doktor Roland Schmiedel. European Pharmacopoeia, 10th edition. 2019.

28. Drevon D, Fursa SR, Malcolm AL. Intercoder Reliability and Validity of WebPlotDigitizer in Extracting Graphed Data. Behav Modif. 2017;41:323-39. doi:10.1177/0145445516673998.

29. Dallal GE. www.randomization.com. 2021. http://www.randomization.com. Accessed 2 Oct 2021.

30. Bittium. https:/www.bittium.com/medical/bittium-faros. Accessed 17 Dec 2019.

31. Krotsetis S, Richards KC, Behncke A, Köpke S. The reliability of the German version of the Richards Campbell Sleep Questionnaire. Nurs Crit Care. 2017;22:247-52. doi:10.1111/nicc.12275.

32. Kim SI, Kim SC, Baek YH, Ok SY, Kim SH. Comparison of ramosetron with ondansetron for prevention of postoperative nausea and vomiting in patients undergoing gynaecological surgery. $\mathrm{Br} \mathrm{J}$ Anaesth. 2009;103:549-53. doi:10.1093/bja/aep209.

33. Anderson LA, Gross JB. Aromatherapy with peppermint, isopropyl alcohol, or placebo is equally effective in relieving postoperative nausea. J Perianesth Nurs. 2004;19:29-35. 
doi:10.1016/j.jopan.2003.11.001.

34. Steyer R, Schwenkmezger P, Notz P. Der Mehrdimensionale Befindlichkeitsfragebogen (MDBF). Göttingen: Hogrefe; 1997.

35. Zegelin A. Zentrale pflegerische Aufgabe: Bewegungsfähigkeit erhalten. Pflegezeitschrift. 2017;70:911. doi:10.1007/s41906-017-0122-0.

36. Sautier LP, Scherwath A, Weis J, Sarkar S, Bosbach M, Schendel M, et al. Erfassung von Arbeitsengagement bei Patienten mit hämatologischen Malignomen: Die psychometrischen Eigenschaften der deutschen Version der Utrecht Work Engagement Scale 9 (UWES-9). Rehabil. 2015;54:297-303.

37. Neumann M, Nagel K, Edelhaeuser F, Scheffer C, Bäuerle K, Wirtz M. Psychometrische Analyse der Messinstrumente "Jefferson Scale for Physician Empathy - Student Version" (JSPE-S) und „Interpersonal Reactivity Index" (IRI). DMW - Dtsch Med Wochenschr. 2012;137 S 03:s-0032-1323395. doi:10.1055/s-0032-1323395.

38. Büssing A, Edelhäuser F, Weisskircher A, Fouladbakhsh JM, Heusser P. Inner Correspondence and Peacefulness with Practices among Participants in Eurythmy Therapy and Yoga: A Validation Study. Evid Based Complement Alternat Med. 2011;2011:1-9. doi:10.1155/2011/329023.

39. MathWorks - Makers of MATLAB and Simulink. https://www.mathworks.com/. Accessed 23 Feb 2021.

40. Harris PA, Taylor R, Thielke R, Payne J, Gonzalez N, Conde JG. Research electronic data capture (REDCap)-a metadata-driven methodology and workflow process for providing translational research informatics support. J Biomed Inform. 2009;42:377-81.

41. R Development Core Team. A language and environment for statistical computing: reference index. Vienna: R Foundation for Statistical Computing; 2010. http://www.R-project.org/. Accessed 23 Feb 2021.

42. Morrison I, Löken LS, Olausson H. The skin as a social organ. Exp Brain Res. 2010;204:305-14. doi:10.1007/s00221-009-2007-y.

43. Ackerley R, Backlund Wasling H, Liljencrantz J, Olausson H, Johnson RD, Wessberg J. Human CTactile Afferents Are Tuned to the Temperature of a Skin-Stroking Caress. J Neurosci. 2014;34:287983. doi:10.1523/JNEUROSCI.2847-13.2014.

44. Walker SC, Cavieres A, Peñaloza-Sancho V, El-Deredy W, McGlone FP, Dagnino-Subiabre A. C-low threshold mechanoafferent targeted dynamic touch modulates stress resilience in rats exposed to chronic mild stress. Eur J Neurosci. 2020;:ejn.14951. doi:10.1111/ejn.14951.

45. Eckstein M, Mamaev I, Ditzen B, Sailer U. Calming Effects of Touch in Human, Animal, and Robotic Interaction-Scientific State-of-the-Art and Technical Advances. Front Psychiatry. 2020;11:555058. doi:10.3389/fpsyt.2020.555058.

46. Qaseem A, Wilt TJ, McLean RM, Forciea MA, Clinical Guidelines Committee of the American College of Physicians. Noninvasive Treatments for Acute, Subacute, and Chronic Low Back Pain: A Clinical Practice Guideline From the American College of Physicians. Ann Intern Med. 2017;166:514-30. 
47. Happe S, Peikert A, Siegert R, Evers S. The efficacy of lymphatic drainage and traditional massage in the prophylaxis of migraine: a randomized, controlled parallel group study. Neurol Sci Off J Ital Neurol Soc Ital Soc Clin Neurophysiol. 2016;37:1627-32.

48. Furlan AD, Giraldo M, Baskwill A, Irvin E, Imamura M. Massage for low-back pain. Cochrane Database Syst Rev. 2015. doi:10.1002/14651858.CD001929.pub3.

49. Chou R, Deyo R, Friedly J, Skelly A, Hashimoto R, Weimer M, et al. Noninvasive Treatments for Low Back Pain. Rockville (MD): Agency for Healthcare Research and Quality (US); 2016. http://www.ncbi.nlm.nih.gov/books/NBK350276/. Accessed 13 Jul 2021.

50. Li Z, Bauer B, Aaberg M, Pool S, Van Rooy K, Schroeder D, et al. Benefits of hand massage on anxiety in preoperative outpatient: A quasi-experimental study with pre- and post-tests. EXPLORE. 2020;:S1550830720303025. doi:10.1016/j.explore.2020.08.016.

51. Brand LR, Munroe DJ, Gavin J. The Effect of Hand Massage on Preoperative Anxiety in Ambulatory Surgery Patients. AORN J. 2013;97:708-17. doi:10.1016/j.aorn.2013.04.003.

52. Bauer BA, Cutshall SM, Wentworth LJ, Engen D, Messner PK, Wood CM, et al. Effect of massage therapy on pain, anxiety, and tension after cardiac surgery: A randomized study. Complement Ther Clin Pract. 2010;16:70-5. doi:10.1016/j.ctcp.2009.06.012.

53. Smith SL, Lux R, Haley S, Slater H, Beachy J, Beechy J, et al. The effect of massage on heart rate variability in preterm infants. J Perinatol Off J Calif Perinat Assoc. 2013;33:59-64.

54. Buttagat $\mathrm{V}$, Eungpinichpong $\mathrm{W}$, Chatchawan $\mathrm{U}, \mathrm{Kharmwan} \mathrm{S}$. The immediate effects of traditional Thai massage on heart rate variability and stress-related parameters in patients with back pain associated with myofascial trigger points. J Bodyw Mov Ther. 2011;15:15-23. doi:10.1016/j.jbmt.2009.06.005.

55. Toro-Velasco C, Arroyo-Morales M, Fernández-de-las-Peñas C, Cleland JA, Barrero-Hernández FJ. Short-Term Effects of Manual Therapy on Heart Rate Variability, Mood State, and Pressure Pain Sensitivity in Patients With Chronic Tension-Type Headache: A Pilot Study. J Manipulative Physiol Ther. 2009;32:527-35. doi:10.1016/j.jmpt.2009.08.011.

56. Yon J-M, Lee O. Effects of Massage on Musculoskeletal Ultrasound and Heart Rate Variability in Middle Age Women of Office Worker. Biomed Sci Lett. 2013;19:55-60.

57. Nenna A, Lusini M, Spadaccio C, Nappi F, Greco SM, Barbato R, et al. Heart rate variability: a new tool to predict complications in adult cardiac surgery. J Geriatr Cardiol JGC. 2017;14:662-8.

58. Kloter E, Barrueto K, Klein SD, Scholkmann F, Wolf U. Heart Rate Variability as a Prognostic Factor for Cancer Survival - A Systematic Review. Front Physiol. 2018;9:623. doi:10.3389/fphys.2018.00623.

59. Ben-Arye E, Portalupi E, Keshet Y, Bonucci M, Can G, Kading Y, et al. Enhancing Palliative Care With Mindful Touch: Impact of a Manual and Movement Therapy Training Program in an International Multidisciplinary Integrative Oncology Setting. J Pain Symptom Manage. 2021;61:229-36. doi:10.1016/j.jpainsymman.2020.08.004.

60. Ozolins L-L, Hörberg U, Dahlberg K. Caring touch - patients' experiences in an anthroposophic clinical context. Scand J Caring Sci. 2015;29:834-42. doi:10.1111/scs.12242. 
61. Field TM, Hernandez-Reif M, Quintino O, Schanberg S, Kuhn C. Elder Retired Volunteers Benefit From Giving Massage Therapy to Infants. J Appl Gerontol. 1998;17:229-39. doi:10.1177/073346489801700210. 